\title{
ACTIVE TRANSPORT OF RB PROTEIN FROM THE NUCLEUS TO THE CYTOPLASM AS ONE OF THE DEVELOPMENT MECHANISMS OF HER2-POSITIVE BREAST CANCER
}

\author{
Artur Kowalik ${ }^{1}$, Janusz Kopczyński ${ }^{1}$, Elżbieta WypiórkiewicZ ${ }^{1}$, Stanisław Góźdź1, \\ RYSZARD MEŻYK ${ }^{1}$, JANUSZ AleKSANDER SiedleCKI ${ }^{2}$
}

\author{
${ }^{1}$ Holycross Cancer Center, Kielce, Poland \\ ${ }^{2}$ Maria Sklodowska-Curie Memorial Cancer Center and Institute of Oncology, Warsaw, Poland
}

\begin{abstract}
HER2-positive breast cancer (HER2+) occurs in approximately 15-20\% of all breast cancers. Biologically this cancer subtype is characterized by an aggressive clinical course (often spread to regional lymph nodes at the time of diagnosis), and after successful treatment high risk of recurrence. Deregulation of the cell cycle is the basis for cancer aggressiveness. The $\mathrm{RB}$ protein is one of the key regulators of the cell cycle. There are only a few published studies on the expression and localization of RB protein in the cells of HER2-positive breast cancer. The aim of this study was to determine whether there are differences in the expression and localization of RB protein in HER2-positive breast cancers compared to breast cancers showing no expression of HER2. We used 50 tissue samples from HER2 positive breast cancer and 21 tissue samples derived from patients with HER 2 negative breast cancer. The RB protein expression was measured by immunohistochemical techniques in tissue microarray format. Cytoplasmic RB expression was observed in 29 out of 50 (58\%) HER2 positive breast cancers. In this group only cytoplasmic expression was observed. There was no case with nuclear expression. In contrast, in the HER2-negative breast cancer control group, in no case $\mathrm{RB}$ expression was observed in the cytoplasm $(0 / 21,0 \%)$. All 21 samples $(100 \%)$ showed expression of $\mathrm{RB}$ protein in the nucleus $(\mathrm{p}<0.0001)$. We can speculate that lack of expression suggests alternative mechanisms in the development of HER2 positive breast cancer. We hypothesize that HER2 overexpression is in some way associated with active transport of $\mathrm{RB}$ protein from the nucleus to the cytoplasm. This may be an indirect mechanism of inactivation of tumor suppressor protein in breast cancer exhibiting overexpression of HER2.
\end{abstract}

Key words: breast cancer, HER2, RB1, active transport.

\section{Introduction}

HER2 gene amplification in breast cancer is associated with shorter disease-free survival and shorter overall survival [1]. Prior to the introduction in 1996 of targeted therapy, breast cancers with HER2+ phenotype very early gave metastases to axillary lymph nodes, bone marrow, liver, lung, ovary and adrenal glands $[2,3]$.
Deregulation of the cell cycle is the basis for cancer aggressiveness [4]. The RB protein is the key regulator of the cell cycle. This protein binds the family of transcription factors $\mathrm{E} 2 \mathrm{~F}$ and releases them as a result of phosphorylation by CDK4/6 in early G1 phase. E2F transcription factors activate the expression of genes essential to carry out the $S$ phase of the cell cycle [4]. Inactivation of the $R B 1$ gene is observed in $20 \%$ of breast cancer cases [5]. In most cancers RB is inactivated di- 
rectly or indirectly. Direct inactivation of the $R B 1$ gene may be due to gene mutation or deletion of parts of chromosome 13 [6]. Indirect inactivation is the result

Table I. Clinicopathological data of the studied group (50 HER2 + patients) and control group (21 HER2- patients)

\begin{tabular}{|c|c|c|}
\hline FEATURE & $\begin{array}{c}\text { HER2 + } \\
(50 \text { PATIENTS) } \\
\text { NuMBER }(\%)\end{array}$ & $\begin{array}{c}\text { HER2- } \\
\text { (21 PATIENTS) } \\
\text { NUMBER }(\%)\end{array}$ \\
\hline \multicolumn{3}{|l|}{ age } \\
\hline$<50$ & $15(30)$ & $5(23)$ \\
\hline$>50$ & $35(70)$ & $16(77)$ \\
\hline \multicolumn{3}{|c|}{ neoadjuvant chemotherapy } \\
\hline+ & $20(40)$ & $4(19)$ \\
\hline- & $30(60)$ & $17(81)$ \\
\hline \multicolumn{3}{|l|}{ clinical stage } \\
\hline 1 & $5(10)$ & $6(27)$ \\
\hline 2 & $20(40)$ & $9(42)$ \\
\hline 3 & $24(48)$ & $5(23)$ \\
\hline 4 & $0(0)$ & $1(4)$ \\
\hline$?$ & $1(2)$ & $1(4)$ \\
\hline \multicolumn{3}{|l|}{$\mathrm{pT}^{*}$} \\
\hline 1 & $17(34)$ & $10(48)$ \\
\hline 2 & $23(46)$ & $8(39)$ \\
\hline 3 & $4(8)$ & $0(0)$ \\
\hline 4 & $4(8)$ & $1(4)$ \\
\hline$?$ & $2(4)$ & $2(9)$ \\
\hline \multicolumn{3}{|l|}{$\mathrm{pN}^{\dagger}$} \\
\hline 0 & $13(26)$ & $14(67)$ \\
\hline 1 & $17(34)$ & $1(4)$ \\
\hline 2 & $10(20)$ & $3(16)$ \\
\hline 3 & $9(18)$ & $1(4)$ \\
\hline$?$ & $1(2)$ & $2(9)$ \\
\hline \multicolumn{3}{|l|}{ grade } \\
\hline 1 & $4(8)$ & $6(27)$ \\
\hline 2 & $24(48)$ & $11(54)$ \\
\hline 3 & $22(44)$ & $4(19)$ \\
\hline \multicolumn{3}{|l|}{ ER } \\
\hline+ & $13(26)$ & $16(77)$ \\
\hline- & $37(74)$ & $5(23)$ \\
\hline \multicolumn{3}{|l|}{$\mathrm{PgR}$} \\
\hline+ & $7(14)$ & $15(73)$ \\
\hline- & $43(86)$ & $6(27)$ \\
\hline \multicolumn{3}{|l|}{ HER2 } \\
\hline $3+$ & $41(82)$ & $0(0)$ \\
\hline $2+(\mathrm{amp}+)$ & $9(8)$ & $0(0)$ \\
\hline $1+$ & $0(0)$ & $2(9.5)$ \\
\hline 0 & $0(0)$ & $19(90.5)$ \\
\hline
\end{tabular}

of its transport from the nucleus to the cytoplasm, where it cannot fulfill its functions. The phosphorylated form of $\mathrm{RB}$ is recognized by the transport protein exportin, which actively moves the RB into the cytoplasm. Excessive phosphorylation of $\mathrm{RB}$ is caused by a hyperactive mutant CDK4 $\left(C D K 4^{R 24 C}\right)$ or by a mitogenic signal which activates CDK 4 [7]. In breast cancer the cytoplasmic location of $\mathrm{RB}$ protein is found in approximately $20 \%$ of cases, and nuclear-cytoplasmic in approximately $40 \%$ [8]. Data on the prognostic significance of RB inactivation in breast cancer are contradictory. So far no connection between the status of $\mathrm{RB}$ and disease-free survival and overall survival has been found $[9,10]$. The aim of this study was to compare the expression and localization of $\mathrm{RB}$ tumor suppressor protein in HER2-positive and HER2-negative breast cancer.

\section{Material and methods}

\section{Characteristics of patients and tissue samples}

Paraffin blocks containing tissue samples from 61 patients who were treated in Holycross Cancer Center (HCC) in 2000-2008 because of invasive breast cancer exhibiting HER 2 overexpression (score $3+$ or $2+$ confirmed by FISH for the presence of HER 2 gene amplification) were selected for the analysis from the archives of the Department of Tumor Pathology HCC. For the final assessment (tested protein, gene amplification and clinicopathological data) material from 50 HER2-positive patients was selected. The remaining 11 cases were excluded due to lack of completeness of the data or insufficient material for immunohistochemical evaluation (Table I). The control group consisted of tissue samples from 21 HER2-negative breast cancer patients (IHC score $1+$ or 0 ) diagnosed and treated surgically during 2008-2009 in the Holycross Cancer Centre in Kielce (Table I).

\section{Construction of TMA blocks}

The TMA block [11] containing HER2+ cases was constructed by compiling two TMA block construction methods previously described in the literature $[12,13]$.

The TMA block containing the control cancer (HER2-) tissue cores (21 cases) was constructed using a commercial kit (Tissue-Tek ${ }^{\circledR}$ Quick-Ray ${ }^{\mathrm{TM}}$ Tissue Microarray System, Sakura Finetek, USA, Inc.) according to the manufacturer's instructions.

\section{Immunohistochemical staining and evaluation criteria}

Immunohistochemical (IHC) staining was performed using standard laboratory procedures and according to the manufacturer's instructions (antibody clone NCL-RB1, Novocastra, diluted $1: 50$ ).

The percentage of stained cells (nuclear or cytoplasmic-cell membrane staining) was assessed in the sec- 
Table II. Comparison of RB protein expression for the study group (HER2+) and control group (HER2-)

\begin{tabular}{lccc}
\hline EXAMINED PROTEIN & HER2 + (50 CASES) & HER2- (21 CASES) & FisHER'S EXACT TEST P VALUE \\
\cline { 2 - 3 } & $\begin{array}{c}\text { DETECTED EXPRESSION } \\
\text { NUMBER OF TUMORS }(\%)\end{array}$ & $\begin{array}{c}\text { DETECTED EXPRESSION } \\
\text { NUMBER OF TUMORS }(\%)\end{array}$ & \\
\hline RB_cytoplasm* & $29(58)$ & $0(0)$ & $<0.0001$ \\
\hline RB_nucleus ${ }^{\dagger}$ & $0(0)$ & $21(100)$ & $<0.0001$ \\
\hline$* R B$ expression evaluated in the cytoplasm, $\dagger R B$ expression evaluated in the nucleus &
\end{tabular}

tions for the analysis of $\mathrm{RB}$ expression. Continuous data type without threshold was used for the Spearman rank correlation analysis. For comparison with literature data the $\mathrm{RB}$ protein expression data were converted to nominal data (Table II) using a threshold value: $\mathrm{RB}$ cytoplasm staining $>10 \%$. If the assessed level of the color reaction was greater than or equal to the threshold, the reaction was treated as positive, and as negative if the level of staining was lower than the threshold. The threshold value ( $>10 \%$ ) was based on the available literature data [10]. When a single case was represented by more than one core in TMA, the average value was calculated. All performed staining reactions were accompanied by control reactions performed simultaneously on appropriate positive and negative control staining cores containing control tissues.

\section{Measurement of HER 2 gene amplification by FISH and evaluation criteria}

HER2 gene status was studied using "PathVysion ${ }^{\circledR}$ HER-2 DNA Probe Kit" (Abbott) and "Paraffin Pretreatment KIT $^{\circledR}$ ", Abbott” as recommended by the manufacturer.

\section{Statistical analysis}

Statistical calculations were performed using MedCalc software version 10.1.3.0 (www.medcalc.be). Fisher test was used to compare RB protein expression between groups and HER2+ and HER2-. Correlations between parameters in the HER2-positive breast cancer group were measured with Spearman's rank correlation coefficient. Statistical significance was accepted when $\mathrm{p}<0.05$.

\section{Results}

$\mathrm{RB}$ protein expression was analyzed in the group of HER2-positive breast cancers (50 cases) and in the control group, i.e. HER2-negative breast cancers (21 cases). Using the TMA method a total of 288 cores cut from donor blocks were analyzed (246 - a group of HER2 + and 42 - a group of HER2-).

In order to check whether the cut cores are representative of the given donor block and thus that the entire tissue microarray made of HER2-positive breast cancers is representative of the material analyzed, we again assessed the status of the HER2/neu gene (FISH). In all cores selected for reassessment, HER2/neu gene amplification was detected (data not shown).

Cytoplasmic RB protein expression was observed in $58 \%$ of HER2-positive invasive breast cancers. In this group, in any case, there was no expression of this protein $(0 \%)$ in the nucleus (Table II and Fig. 1). In the control HER2-negative breast cancer group, RB expression was not observed in the cytoplasm. In contrast, all $(100 \%)$ tumors in this group showed expression of $\mathrm{RB}$ protein in the nucleus (Table II and Fig. 2).

Then, in order to examine whether there were any relationships between expression of $\mathrm{RB}$ in the cytoplasm in HER2-positive breast cancers and the available clinicopathological data [age at time of surgery, status of ER and PgR, tumor size (pT), grade (G), clinical stage (stage), neoadjuvant therapy treatment, degree of spread to regional lymph nodes $(\mathrm{pN})$ ] Spearman's rank correlation coefficient test was used. However, it revealed no statistically significant $(\mathrm{p}<0.05)$ correlation.

\section{Discussion}

HER2-positive breast cancer is a very aggressive disease, rapidly causing distant metastases [1]. The aggressiveness of cancer is in most cases due to the loss of control over the cell cycle. RB protein is one of the key regulators of the cell cycle [4]. To the best of our knowledge, there is little published work on RB protein expression and localization in HER2-positive

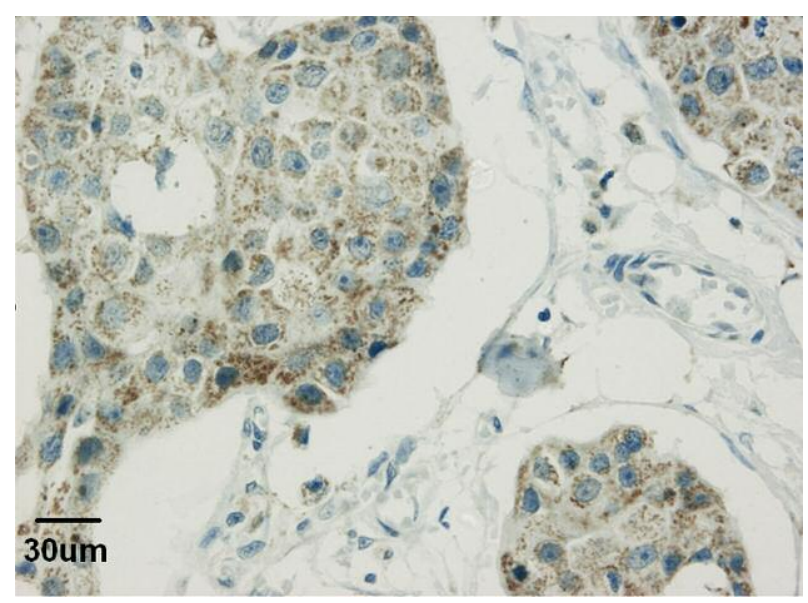

Fig. 1. Expression of RB in the cytoplasm in HER2-positive breast cancer measured by IHC (magnification $400 \times$ ) 


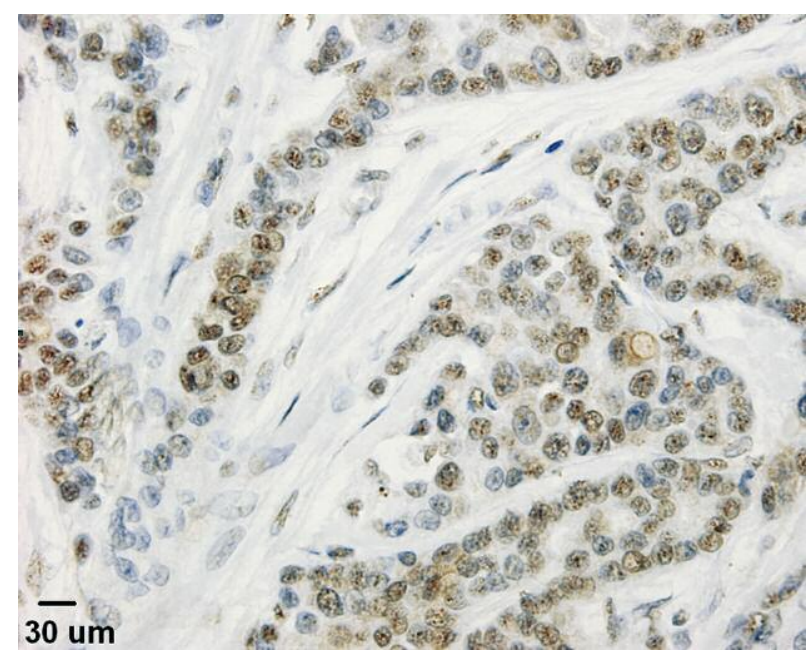

Fig. 2. Expression of RB in the cell nucleus in HER2-negative breast cancer measured by IHC (magnification $400 \times$ )

breast cancer so we decided to examine the location of this protein in these tumors in comparison to HER2negative breast cancers. The analysis was conducted using immunohistochemistry in TMA format.

In this study RB expression was not detected in the nucleus in any of the cases of HER2-positive breast cancer (Table II). There are some discrepancies between our results and the recently published study of Stefansson et al. [14]. The main objective of that study was to evaluate basal/triple-negative invasive breast cancers. In that study, the authors analyzed 303 cases of invasive breast cancer. Only 21 cases showed overexpression of HER2; the authors found in most of them
$\mathrm{RB}$ expression in the nucleus. Unfortunately, the authors did not specify whether they analyzed the expression of RB in the cytoplasm. In addition, the difference may be due to the use of another antibody clone [14]. In another study conducted by Pinto et al., HER2positive breast cancer cases (IHC $3+$ and $2+$ ) showed no relation to the location of $\mathrm{RB}$ in the nucleus. Also that study did not analyze the expression of $\mathrm{RB}$ in the cytoplasm [10]. In the present study we did not find any correlation between RB expression in the cytoplasm and available clinicopathological data, which could indicate the independence of this event in biology of HER2-positive breast cancer.

Lack of $\mathrm{RB}$ protein in the nucleus may be due to gene mutations, loss of heterozygosity ( $\mathrm{LOH}$ ), or methylation of the $R B 1$ gene promoter [6] and may contribute to loss of cell cycle control [4].

Recently, Jiao et al. described the mechanism of active $\mathrm{RB}$ protein transport from the nucleus to the cytoplasm mediated by exportin-1 [7]. This mechanism may be induced by a mutated cyclin-dependent kinase 4 CDK4 (Arg24Cys) or activation of the correct form of $\mathrm{CDK} 4$ through mitogenic signals induced by receptor tyrosine kinase $[7,8]$. In the case of this study, the source of the mitogenic signal may be HER2 (Fig. 3).

For comparison, in tumors with HER2-negative breast cancers, expression of $\mathrm{RB}$ in the cell nucleus was detected in all cases ( $\mathrm{p}<0.0001$, Table II, Fig. 2). The cytoplasmic expression of RB was detected in $29(58 \%)$ HER2-positive breast cancers (Table II and Fig. 1). In HER2-negative breast cancers cytoplasmic expression was not detected in any case $(\mathrm{p}<0.0001$, Table II,

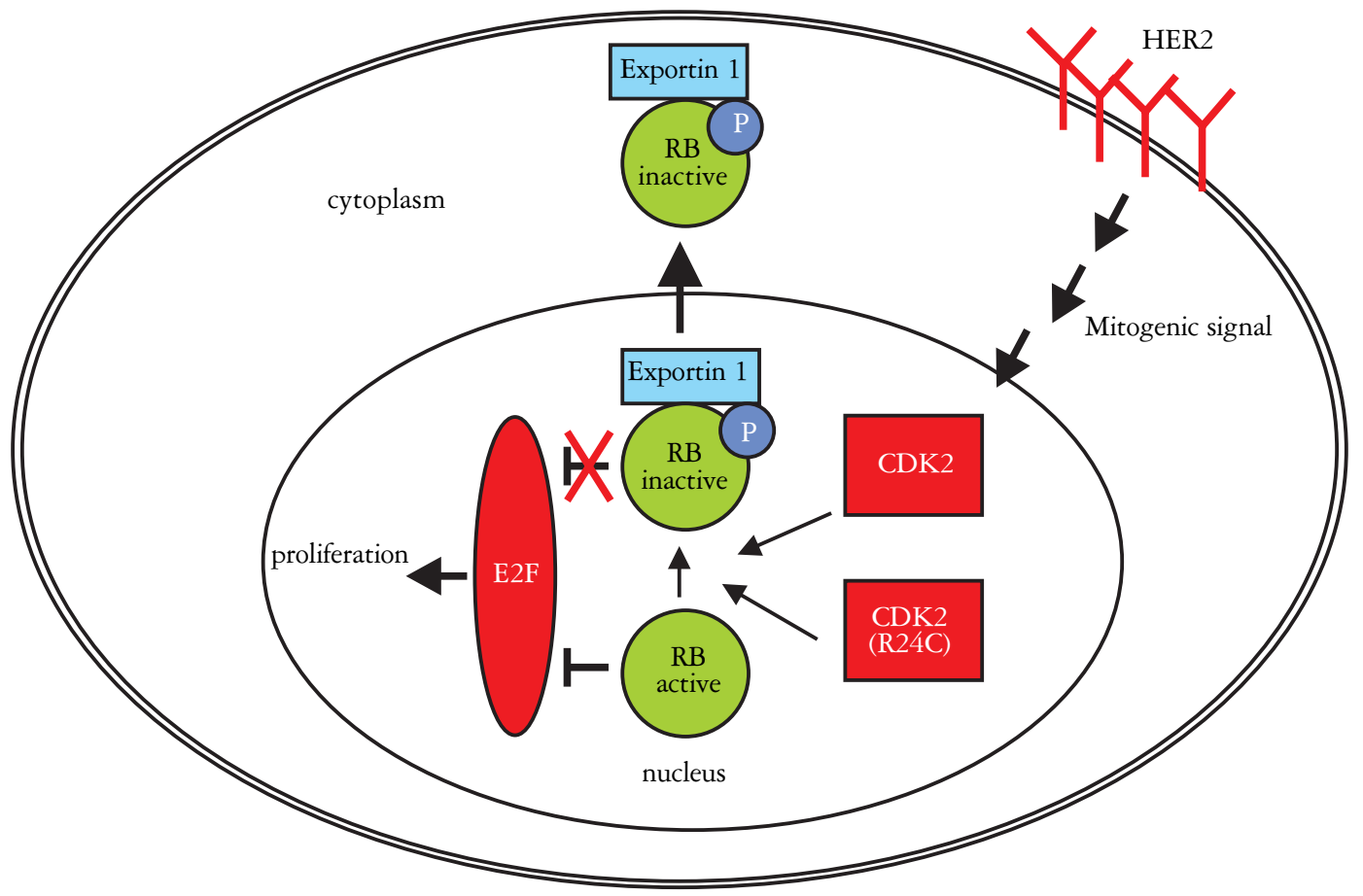

Fig. 3. Mechanisms of active transport of RB from the nucleus to the cytoplasm 
Fig. 2). This observation may support the hypothesis of active transport of RB from the nucleus to the cytoplasm as a result of HER2 overexpression. Lack of $\mathrm{RB}$ in the nucleus promotes deregulation of the cell cycle and can lead to higher incidence of aneuploidy and abnormalities in chromosome structure [15], i.e. global genomic instability in HER2-positive breast cancer $[16]$.

Additional data about the transport of RB from the nucleus to the cytoplasm were provided by a study using an animal model. In that study, Japanese researchers demonstrated the expression of phosphorylated RB (p$\mathrm{RB}$ ) in the cytoplasm of advanced thyroid cancer compared to adenomas or carcinomas less advanced, which showed expression only in the nucleus. The authors drew the conclusion that the displacement of $\mathrm{RB}$ proteins from the nucleus to the cytoplasm is one of the mechanisms of the development of aggressive thyroid cancer [17].

A similar subcellular localization was observed in the case of expression of MYC in breast cancer tumor cells. It should be noted that expression of MYC protein was detected both in the nucleus and the cytoplasm [18]. The available data [19] indicate that the ratio of expression detected in the nucleus to the expression detected in the cytoplasm is greater in normal cells and in invasive cancer cells decreases in favor of cytoplasmic expression. In the cited study the authors unfortunately do not provide the status of HER2 in the breast carcinoma cases tested [19]. Despite the known functions of MYC and RB in the cell nucleus, in the cytoplasm their role still remains unclear.

Further evidence supporting the hypothesis of active $\mathrm{RB}$ transport activated by HER2 overexpression is provided by preclinical and clinical studies on the mechanisms of action of anti-estrogen therapies. Preclinical studies have shown that anti-estrogen therapy stops proliferation and induces apoptosis. These two processes are related to the fact that blocking the action of ER leads to reduced expression of cyclin D1 and MYC. The result is a lack of phosphorylation of RB and cell cycle arrest [20]. Patients with HER2+/HR + breast cancer show a weaker response to anti-estrogen therapy compared to patients with HER2-/HR + breast cancer. The addition of trastuzumab to anti-estrogen therapy of HER $2+/ \mathrm{HR}+$ breast cancer patients improves outcomes [21]. This can be explained by blocking the activity of HER2, resulting in blockade of the active transport of RB from the nucleus to the cytoplasm, which restores its tumor suppressor activity. Unfortunately the treatment results are not as good as for the administration of trastuzumab combined with chemotherapy [21]. This might apply to cases where there was a deletion of the gene encoding the protein RB.

The detected lack of expression of RB in the nucleus in all studied cases may suggest alternative mechanisms for the development of HER2-positive breast cancer.
These results suggest that HER2 overexpression may be associated with active transport of RB protein from the nucleus to the cytoplasm. This may be an indirect mechanism of inactivation of tumor suppressor protein in HER2-positive breast cancer cases, which may be relevant to identify new targets for targeted therapy.

The authors declare that they have no conflicts of interest. All experiments were conducted in accordance with Polish law and the guidelines established by the Declaration of Helsinki.

\section{References}

1. Slamon DJ, Clark GM, Wong SG, et al. Human breast cancer: correlation of relapse and survival with amplification of the HER2/neu oncogene. Science 1987; 235: 177-182.

2. Baselga J, Tripathy D, Mendelsohn J, et al. Phase II study of weekly intravenous recombinant humanized anti-p185HER2 monoclonal antibody in patients with HER2/neu-overexpressing metastatic breast cancer. J Clin Oncol 1996; 14: 737-744.

3. Spigel DR, Burstein HJ. HER2 overexpressing metastatic breast cancer. Curr Treat Options Oncol 2002; 3: 163-174.

4. Hanahan D, Weinberg RA. The hallmarks of cancer. Cell 2000; 100: $57-70$.

5. Fung YK, T'Ang A. The role of the retinoblastoma gene in breast cancer development. Cancer Treat Res 1992; 61:59-68.

6. Burkhart DL, Sage J. Cellular mechanisms of tumour suppression by the retinoblastoma gene. Nat Rev Cancer 2008; 8: 671682.

7. Jiao W, Datta J, Lin HM, et al. Nucleocytoplasmic shuttling of the retinoblastoma tumor suppressor protein via $\mathrm{Cdk}$ phosphorylation-dependent nuclear export. J Biol Chem 2006; 281: 38098-38108.

8. Jiao W, Lin HM, Datta J, et al. Aberrant nucleocytoplasmic localization of the retinoblastoma tumor suppressor protein in human cancer correlates with moderate/poor tumor differentiation. Oncogene 2008; 27: 3156-3164.

9. Oliveira AM, Ross JS, Fletcher JA. Tumor suppressor genes in breast cancer: the gatekeepers and the caretakers. Am J Clin Pathol 2005; 124 Suppl:S16-28.

10. Pinto AE, Andre S, Laranjeira C, et al. Correlations of cell cycle regulators (p53, p21, pRb and $\mathrm{mdm} 2)$ and c-erbB-2 with biological markers of proliferation and overall survival in breast cancer. Pathology 2005; 37: 45-50.

11. Kononen J, Bubendorf L, Kallioniemi A, et al. Tissue microarrays for high-throughput molecular profiling of tumor specimens. Nat Med 1998; 4: 844-847.

12. Chen N, Zhou Q. Constructing tissue microarrays without prefabricating recipient blocks: a novel approach. Am J Clin Pathol 2005; 124: 103-107.

13. Pires AR, Andreiuolo Fda M, de Souza SR. TMA for all: a new method for the construction of tissue microarrays without recipient paraffin block using custom-built needles. Diagn Pathol 2006; $1: 14$.

14. Stefansson OA, Jonasson JG, Olafsdottir K, et al. CpG island hypermethylation of BRCA1 and loss of $\mathrm{pRb}$ as co-occurring events in basal/triple-negative breast cancer. Epigenetics 2011; 6: 638-649.

15. van Deursen JM. Rb loss causes cancer by driving mitosis mad. Cancer Cell 2007; 11: 1-3.

16. Ellsworth RE, Ellsworth DL, Patney HL, et al. Amplification of HER2 is a marker for global genomic instability. BMC Cancer 2008; 8:297. 
17. Ago K, Saegusa Y, Nishimura J, et al. Involvement of glycogen synthase kinase-3beta signaling and aberrant nucleocytoplasmic localization of retinoblastoma protein in tumor promotion in a rat two-stage thyroid carcinogenesis model. Exp Toxicol Pathol 2010; 62: 269-280.

18. Liao DJ, Dickson RB. c-Myc in breast cancer. Endocr Relat Cancer 2000; 7: 143-164

19. Blancato J, Singh B, Liu A, et al. Correlation of amplification and overexpression of the c-myc oncogene in high-grade breast cancer: FISH, in situ hybridisation and immunohistochemical analyses. Br J Cancer 2004; 90: 1612-1619.

20. Musgrove EA, Sutherland RL. Biological determinants of endocrine resistance in breast cancer. Nat Rev Cancer 2009; 9: 631643

21. Prat A, Baselga J. The role of hormonal therapy in the management of hormonal-receptor-positive breast cancer with coexpression of HER2. Nat Clin Pract Oncol 2008; 5: 531-542.

\section{Address for correspondence}

\section{Artur Kowalik}

Holycross Cancer Center

Artwińskiego 3

25-374 Kielce, Poland

e-mail: artur_kowalik@yahoo.com

tel. +48413674259

fax +48413674260 